\title{
MSCT versus CBCT: evaluation of high-resolution acquisition modes for dento-maxillary and skull-base imaging
}

\author{
Jean-Philippe Dillenseger • Jean-François Matern • Catherine-Isabelle Gros • \\ Fabien Bornert • Christian Goetz • Jean-Marie Le Minor • \\ André Constantinesco • Philippe Choquet
}

Received: 23 July 2014 /Revised: 19 August 2014 / Accepted: 8 September 2014

(C) European Society of Radiology 2014

\begin{abstract}
Objectives Our aim was to conduct a quantitative and qualitative evaluation of high-resolution skull-bone imaging for dentistry and otolaryngology using different architectures of recent X-ray computed tomography systems.

Material and methods Three multi-slice computed tomography (MSCT) systems and one Cone-beam computed tomography (CBCT) system were used in this study. All apparatuses were tested with installed acquisition modes and proprietary reconstruction software enabling high-resolution bone imaging. Quantitative analyses were performed with small fields of view with the preclinical vmCT phantom, which permits to measure spatial resolution, geometrical accuracy, linearity and homogeneity. Ten operators performed visual qualitative
\end{abstract}

J.-P. Dillenseger · J.-F. Matern $\cdot$ C. Goetz $\cdot$ A. Constantinesco $\cdot$

P. Choquet

Imagerie Préclinique-UF6237, Pôle d'imagerie, Hôpitaux

Universitaires de Strasbourg, Strasbourg, France

J.-P. Dillenseger $\cdot$ C. Goetz · J.-M. Le Minor $\cdot$ P. Choquet Icube, équipe MMB, CNRS, Université de Strasbourg, Strasbourg, France

J.-P. Dillenseger · J.-F. Matern • C.-I. Gros • F. Bornert • C. Goetz • J.-M. Le Minor · P. Choquet

Fédération de Médecine Translationnelle de Strasbourg, Faculté de Médecine, Université de Strasbourg, Strasbourg, France

C.-I. Gros $•$ F. Bornert

Faculté de Chirurgie Dentaire, Université de Strasbourg, Strasbourg, France

J.-M. Le Minor

Institut d'Anatomie Normale, Université de Strasbourg, Strasbourg, France

P. Choquet $(\triangle)$

Imagerie Préclinique, Biophysique et Médecine Nucléaire, Hôpital de Hautepierre, 1 Av. Molière, 67098 Strasbourg Cedex, France

e-mail: pchoquet@unistra.fr analyses on the vmCT phantom images, and on dry human skull images.

Results Quantitative analysis showed no significant differences between protocols in terms of linearity and geometric accuracy. All MSCT systems present a better homogeneity than the CBCT. Both quantitative and visual analyses demonstrate that $\mathrm{CBCT}$ acquisitions are not better than the collimated helical MSCT mode.

Conclusion Our results demonstrate that current highresolution MSCT protocols could exceed the performance of a previous generation CBCT system for spatial resolution and image homogeneity.

Key Points

- Quantitative evaluation is a prerequisite for comparison of imaging equipment.

- Bone imaging quality could be objectively assessed with a phantom and dry skull.

- The current MSCT shows better image quality than a dental CBCT system.

- CBCT remains a work-in-progress technology.

Keywords Cone-beam computed tomography $\cdot$ Multi-slice computed tomography · Bone - Quantitative evaluation . Radiological phantoms

$\begin{array}{ll}\text { Abbreviations } \\ \text { CBCT } & \text { Cone-beam computed tomography } \\ \text { CT } & \text { Computed tomography } \\ \text { FBP } & \text { Filtered back projection } \\ \text { FOV } & \text { Field of view } \\ \text { FPD } & \text { Flat panel detector } \\ \text { MSCT } & \text { Multi-slice computed tomography } \\ \text { MTF } & \text { Modulation transfer function } \\ \text { ROI } & \text { Regions of interest } \\ \text { SD } & \text { Standard deviation } \\ \text { VOI } & \text { Volumes of interest }\end{array}$




\section{Introduction}

The diagnosis of thin dento-maxillary and skull-base lesions requires high-definition bone imaging. Nowadays, $\mathrm{X}$-ray computed tomography (CT) is the best available technical issue for studying tiny bone structures [1]. Over the last decade, manufacturers designed polyvalent multi-slice CT systems (MSCT) in order to reduce acquisition time, especially for cardiology, vascular, whole body and emergency imaging [2-4]. There are various technological approaches aimed at reducing acquisition times; for instance, increasing the number of rows for incremental acquisition or proposing dual-source imaging for dual helical acquisitions. Today, however, higher spatial resolution imaging is no longer a priority relative to the search for temporal resolution and radiation safety. Nevertheless, clinical applications concerning dentomaxillar and temporal bone regions [1] need higher resolution, as for instance when searching for a fistula tract in maxillar osteomyelitis, or superior canal dehiscence. More recently, a lower-cost specific architecture was designed for high-resolution skull-bone imaging using a flat panel detector (FPD) called cone-beam CT (CBCT) [5, 6]. We compare in this study three MSCT and one CBCT, from different manufacturers, that have been installed in our University Hospital since 2011. The purpose of this study is to evaluate linearity, geometric accuracy, homogeneity and spatial resolution for these apparatuses using a phantom adapted to small field of view (FOV) and high spatial resolution [7]. Each CT system was tested with protocols used in our clinical routine. To complete the quantitative analysis, a human dry skull was examined under the same conditions in order to allow for a visual qualitative evaluation.

\section{Material and methods}

\section{CT systems and acquisition protocols}

The MSCT instruments were: Somatom Flash CT (Siemens Medical, Erlangen, Germany); Discovery CT750HD (GE Healthcare, Milwaukee, USA); and Aquilion One 320 (Toshiba Medical Systems, Nasu, Japan). The CBCT was a Newtom 5G (QR s.r.l, Verona, Italy). All apparatuses were tested with modes that enable high-resolution bone imaging; they were all up-to-date in terms of hardware and software at the time of the study. We decided to work only with filtered back-projection (FPB) reconstruction algorithms, in order to make a comparison between all systems possible. Reconstructions were all done using the proprietary software. The characteristics of the CT systems and protocol acquisitions are

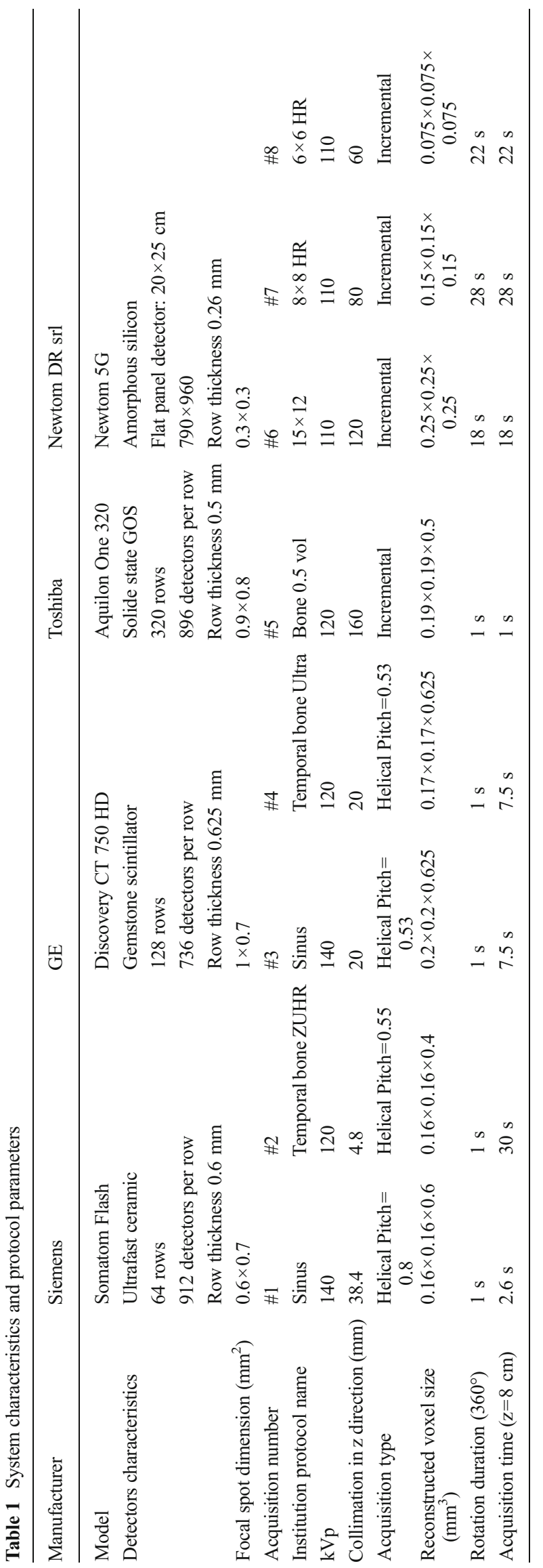




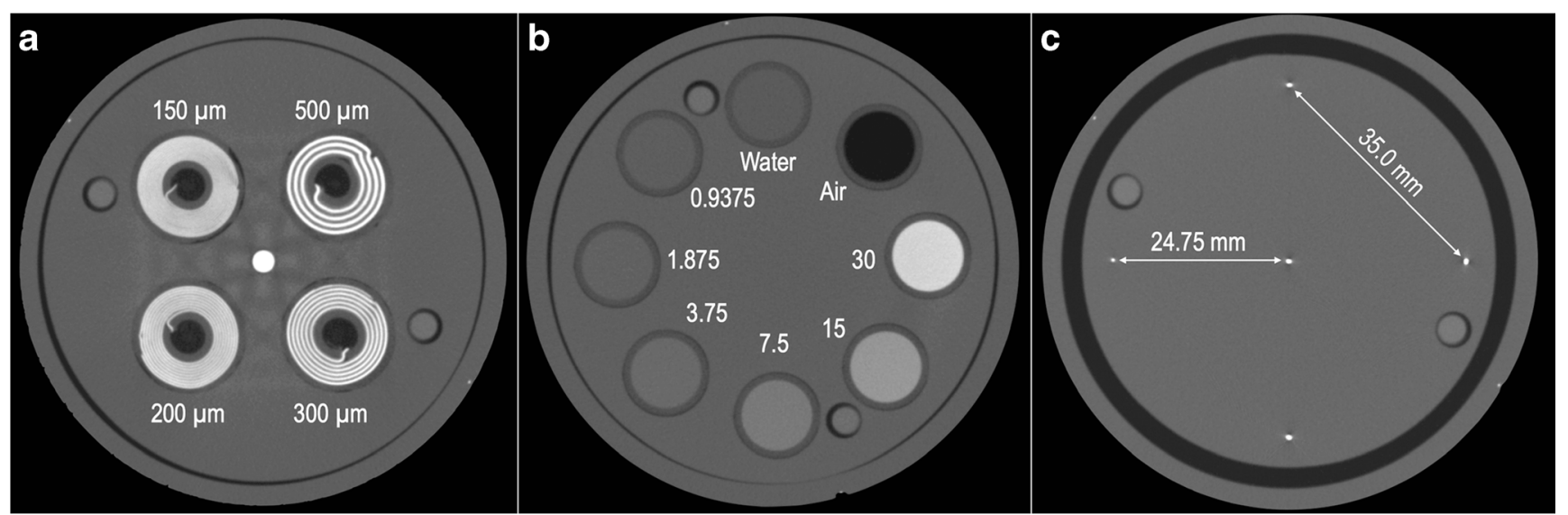

Fig. 1 Separate modular sections of the vmCT phantom acquired on a preclinical micro-CT system (eXplore CT 120, GE Healthcare, Waukesha, WI, USA). Resolution coil plate (a), linearity plate with iodine concentration listed in $\mathrm{mg}^{-\mathrm{ml}^{-1}}$ (b), Geometric accuracy plate with copper wire (c)

summarized in Table 1. Each acquisition has been numbered (from \#1 to \#8); this identification number will be used in the following for further reference. For MSCT, we used the proposed standard and high-resolution bone protocols. These two protocols were not available for the Toshiba system and we used the best-resolved acquisition protocol offered by the manufacturer.

\section{Phantom}

The vmCT (Shelley medical technologies, Toronto, Canada) is a phantom [6] consisting of separated modular sections, each designed to evaluate one particular aspect of image quality (resolution, geometric accuracy, linearity, uniformity and noise). The sections are held together inside an acrylic tube with an inner diameter of $63 \mathrm{~mm}$ and an outer diameter of $70 \mathrm{~mm}$; the total length of the phantom is $54 \mathrm{~mm}$ (Fig. 1). These dimensions are adapted to the use of a small FOV especially for the CBCT acquisition \#8 (Table 1).

\section{Spatial resolution}

The resolution coil plate of the phantom (Fig. 1) provides a visual qualitative measurement of the spatial resolution of the system. Embedded in the polycarbonate plastic plate of $4.8 \mathrm{~mm}$ thickness, there are four alternated aluminium and mylar sheets spiral coils, with layer thicknesses of 150, 200,
300 and $500 \mu \mathrm{m}$, corresponding to 3.3, 2.5, 1.67 and 1 line pairs per $\mathrm{mm}\left(\mathrm{lp} . \mathrm{mm}^{-1}\right)$, respectively. The standard deviation (SD) in four volumes of interest (VOI) of $1.5 \times 1.5 \times 1.5 \mathrm{~mm}^{3}$ placed on each coil (top, bottom, left and right) were measured and corrected by the SD of the phantom uniform region. For the quantitative spatial resolution analysis, the modulation transfer function (MTF) values were calculated for each coil thickness using the average SD and the mean absolute difference $\mathrm{CT}$ values measured for aluminum and plastic $[8,9]$. The reference value (MTF 100) was determined from the CT number for pure aluminum $\left(\mathrm{CT}_{\mathrm{Al}}\right)$ and plastic $\left(\mathrm{CT}_{\text {plastic }}\right)$, according to formula (1) [9].

$\mathrm{MTF} 100=\frac{\left|\mathrm{CT}_{\mathrm{A} 1}-\mathrm{CT}_{\text {plastic }}\right|}{2}$

A subjective blinded visual analysis was conducted by ten trained $\mathrm{CT}$ users (radiologists and technologists) to qualitatively classify the different acquisitions. For each acquisition, a semiquantitative visual scale using three subscores evaluating spatial resolution, blurring and image artefacts, was applied (Table 2). The different acquisitions were ranked from these results. This blind analysis was performed using the free software Osirix (www.osirixviewer.com).
Table 2 Phantom analysis scoring

$\begin{array}{lllllll}\text { Phantom analysis scale } & & & & & \\ \text { Spiral coil }(500 \mu \mathrm{m}) & \text { non visible } & 0 & \text { lightly visible } & 1 & \text { clearly visible } & 2 \\ \text { Spiral coil }(300 \mu \mathrm{m}) & \text { non visible } & 0 & \text { lightly visible } & 1 & \text { clearly visible } & 2 \\ \text { Spiral coil }(200 \mu \mathrm{m}) & \text { non visible } & 0 & \text { lightly visible } & 1 & \text { clearly visible } & 2 \\ \text { Spiral coil }(150 \mu \mathrm{m}) & \text { non visible } & 0 & \text { lightly visible } & 1 & \text { clearly visible } & 2 \\ \text { Blurring and artifacts } & \text { detrimental } & 0 & \text { troublesome } & 1 & \text { non visible } & 2\end{array}$


Table 3 Human skull analysis scoring

\begin{tabular}{|c|c|c|c|c|c|c|}
\hline \multicolumn{7}{|c|}{ Dento-maxillar region analysis scale } \\
\hline Dental and peridental & low quality & 0 & convenient & 1 & high quality & 2 \\
\hline Alveolar bone & low quality & 0 & convenient & 1 & high quality & 2 \\
\hline Blurring and artifacts & detrimental & 0 & troublesome & 1 & non visible & 2 \\
\hline \multicolumn{7}{|c|}{ Temporal region analysis scale } \\
\hline Petrous bone & low quality & 0 & convenient & 1 & high quality & 2 \\
\hline Ear & low quality & 0 & convenient & 1 & high quality & 2 \\
\hline Blurring and artifacts & detrimental & 0 & troublesome & 1 & non visible & 2 \\
\hline
\end{tabular}

\section{Geometric accuracy}

Four beads were placed $35 \mathrm{~mm}$ apart at the corners of an ideal square centred in the geometric accuracy section (Fig. 1), and one bead was placed at the centre of the square $(24.75 \mathrm{~mm}$ from the other four). The distance between two neighbouring beads was measured between the beads centres on native slices. The differences between the known physical distances and the measured distances were expressed in $\%$ and the mean distance error of each acquisition was compared to its pixel size.

\section{Linearity}

The linearity of the system was determined by the linearity plate, which consisted of vials of increasing iodine concentrations of $0.9375,1.875,3.75,7.5,15,30 \mathrm{mg} . \mathrm{ml}^{-1}$ [6]. The mean signal intensity and standard deviation for each iodine concentration were measured in HU in a $3 \times$ $3 \times 3 \mathrm{~mm}^{3}$ VOI placed manually at the centre of each vial.
The relationship between signal intensity and iodine concentration was determined by linear regression analysis (Microsoft Excel, Redmond, Washington, USA).

\section{Uniformity}

Four peripheral regions of interest (ROI) and one central ROI of $3 \times 3 \times 1 \mathrm{~mm}^{3}$ were drawn on the uniformity section in order to quantitatively assess the variation in signal intensity from the centre to the periphery. The average differences in signal intensity between the peripheral and the central regions, as well as the average measured SD measured in the five ROIs were used to calculate the uniformity-to-noise ratio. The total measured noise can be considered as a quadrature summation of photon noise and system noise [10].

\section{Human skull-bone analysis}

A human dry skull was loaned from the collection of the Institute of Normal Anatomy of the Strasbourg Faculty of
Fig. 2 Modulation transfer function (MTF) of each protocol (\#1 to \#8; see Table 1) measured from the resolution coil plate

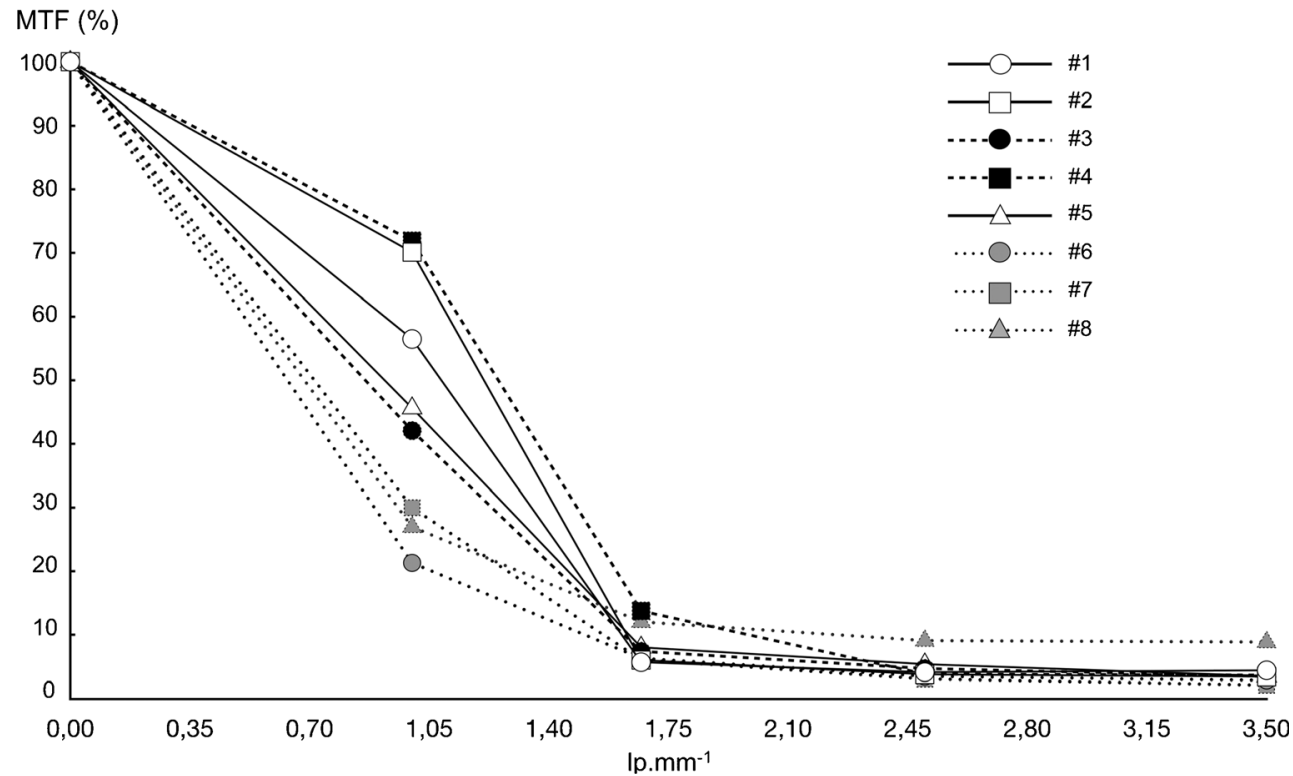


Fig. 3 Axial slices through the resolution plates for different protocols (\#1 to \#8; see Table 1).

Window level: 700; window width: 4000
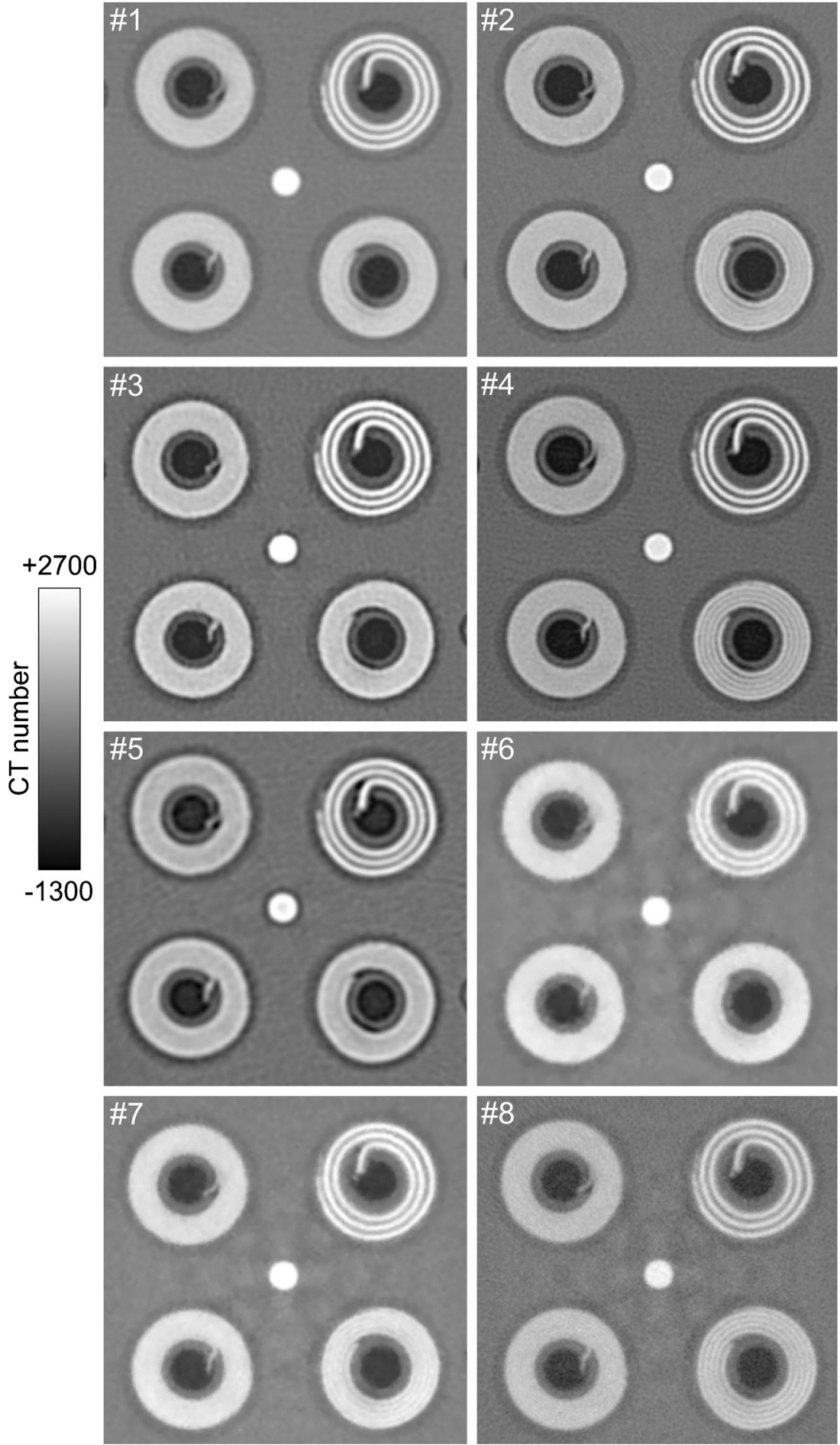

Medicine [11], in order to test different protocols on real bone architecture. A blind visual analysis was conducted by ten different trained CT users (radiologists and technologists) for classifying the different acquisitions. In order to be comparable over the different systems, a dental maxillary region and a temporal region were examined to perform this visual qualitative analysis. For each acquisition, a semiquantitative visual scale, using three subscores that evaluated anatomical structures, blurring and image artefacts, was applied (Table 3 ). The scores were adjusted to ten and the different acquisitions were 
Table 4 Phantom and human dry skull scoring results

\begin{tabular}{|c|c|c|c|c|c|c|c|c|}
\hline \multirow[t]{2}{*}{ Rank } & \multicolumn{2}{|l|}{ Radiologic phantom } & \multicolumn{2}{|c|}{ Dento-maxillary region } & \multicolumn{2}{|c|}{ Temporal bone region } & \multicolumn{2}{|l|}{ Mean } \\
\hline & Score / $10(n=10)$ & Acq. number & Score / $10(n=10)$ & Acq. number & Score / $10(n=10)$ & Acq. number & Score / 10 & Acq. number \\
\hline 1 & 5.6 & $\# 4$ & 8.8 & $\# 2$ & 8.9 & $\# 2$ & 7.6 & $\# 2$ \\
\hline 2 & 5.1 & $\# 2$ & 7.7 & $\# 4$ & 8.4 & $\# 4$ & 7.2 & $\# 4$ \\
\hline 3 & 4.3 & $\# 8$ & 6.9 & $\# 8$ & 5.2 & $\# 3$ & 5.0 & $\# 8$ \\
\hline 4 & 3.8 & $\# 1$ & 5.0 & $\# 7$ & 4.5 & $\# 1$ & 4.2 & $\# 1$ \\
\hline 5 & 3.2 & $\# 3$ & 4.3 & $\# 1$ & 4.0 & $\# 5$ & 3.7 & $\# 3$ \\
\hline 6 & 2.8 & $\# 7$ & 3.3 & $\# 6$ & 3.8 & $\# 8$ & 3.5 & $\# 7$ \\
\hline 7 & 2.7 & $\# 5$ & 2.7 & $\# 3$ & 2.7 & $\# 7$ & 2.8 & $\# 5$ \\
\hline 8 & 1.6 & $\# 6$ & 1.7 & $\# 5$ & 1.0 & \#6 & 2.0 & $\# 6$ \\
\hline
\end{tabular}

ranked from these results. This blind analysis was performed using the free software OsiriX (www.osirix-viewer.com).

\section{Results}

Spatial resolution

The in-plane MTF of each system was based on the analysis of the reconstructed images of the coils. For quantitative analysis, the MTF at 1, 1.67, 2,50, $3.50 \mathrm{lp} \cdot \mathrm{mm}^{-1}$ of each acquisition are summarized in Fig. 2.

The visual qualitative analysis allowed the visualisation of the $500 \mu \mathrm{m}$ coil for each acquisition protocol, the $300 \mu \mathrm{m}$ coil was seen for the \#2, \#3, \#4,\#7 and \#8 acquisitions, the $200 \mu \mathrm{m}$ coil plate was only seen in the acquisition \#8, and the $150 \mu \mathrm{m}$ coil plate was never seen (Fig. 3). Scores and rankings issued from different acquisitions are given in Table 4. These rankings match the quantitatively observed results (Fig. 2).
Geometric accuracy

For each acquisition, the measured errors were smaller than the dimensions of the pixels (Table 4), meaning that the geometric accuracy is efficient for all acquisitions and systems.

\section{Linearity}

The linearity of the system was determined using the measured CT number in iodine solutions at various concentrations (Fig. 1). Linear correlation coefficients $\mathrm{R}^{2}$ between measured CT number and iodine concentration are given in Table 5. The $\mathrm{R}^{2}$ correlation coefficient value is comprised between 0.9809 and 0.9931 , which shows that all systems are highly linear over the range of signal intensities encountered.

\section{Uniformity}

Uniformity of the system's signal response was measured on the polycarbonate uniformity section. The average difference in signal intensity values between peripheral and central regions is reported in Table 4. Contrary to CBCT protocols (\#6

Table 5 Geometric accuracy, linearity and homogeneity results for each protocol (\#1 to \#7; see Table 1)

\begin{tabular}{|c|c|c|c|c|c|c|c|c|}
\hline Acquisition number & $\# 1$ & $\# 2$ & \#3 & $\# 4$ & $\# 5$ & $\# 6$ & $\# 7$ & $\# 8$ \\
\hline \multicolumn{9}{|l|}{ Geometric accuracy } \\
\hline $\begin{array}{l}\text { Mean measurement } \\
\text { error }(\mathrm{mm})\end{array}$ & 0.08 & 0.04 & 0.07 & 0.10 & 0.11 & 0.02 & 0.05 & 0.011 \\
\hline Pixel size (mm) & 0.16 & 0.16 & 0.20 & 0.17 & 0.19 & 0.25 & 0.15 & 0.075 \\
\hline \multicolumn{9}{|l|}{ Linearity } \\
\hline $\mathrm{R}^{2}$ & 0.9826 & 0.9896 & 0.9916 & 0.9884 & 0.9709 & 0.9931 & 0.9893 & 0.9924 \\
\hline \multicolumn{9}{|l|}{ Homogeneity } \\
\hline Uniformity (SD) in CT value & $5.5(1.29)$ & $11.5(7)$ & $1.75(1.28)$ & $2.5(2.38)$ & $6.53(2.51)$ & $28.5(24.4)$ & $35.03(23.03)$ & $33.52(31.06)$ \\
\hline Noise (SD) in CT value & $43(6.52)$ & $27.2(1.1)$ & $22(1.41)$ & $33.2(1.79)$ & $25.83(2.86)$ & $16.18(4.04)$ & $22.56(3.13)$ & $34.84(1.94)$ \\
\hline
\end{tabular}


and \#8), we note that for MSCT protocols (\#1 to \#5) the average differences in signal intensity values between centre and periphery were all lower than the measured noise-signal intensity values. This means that the MSCT systems present a better uniformity than the CBCT system. Radial signal profiles of the uniformity section illustrate this point (Fig. 4).
Human skull-bone

Figure 5 shows a slice at the same position of the dental region obtained with each protocol (\#1 to \#8; see Table 1). Figure 6 presents axial slices through the right temporal bone (\#1 to \#8; see Table 1). Scores and ranking issued from these different
Fig. 4 Radial signal profiles of the uniformity section for each protocols (\#1 to \#8; see Table 1)
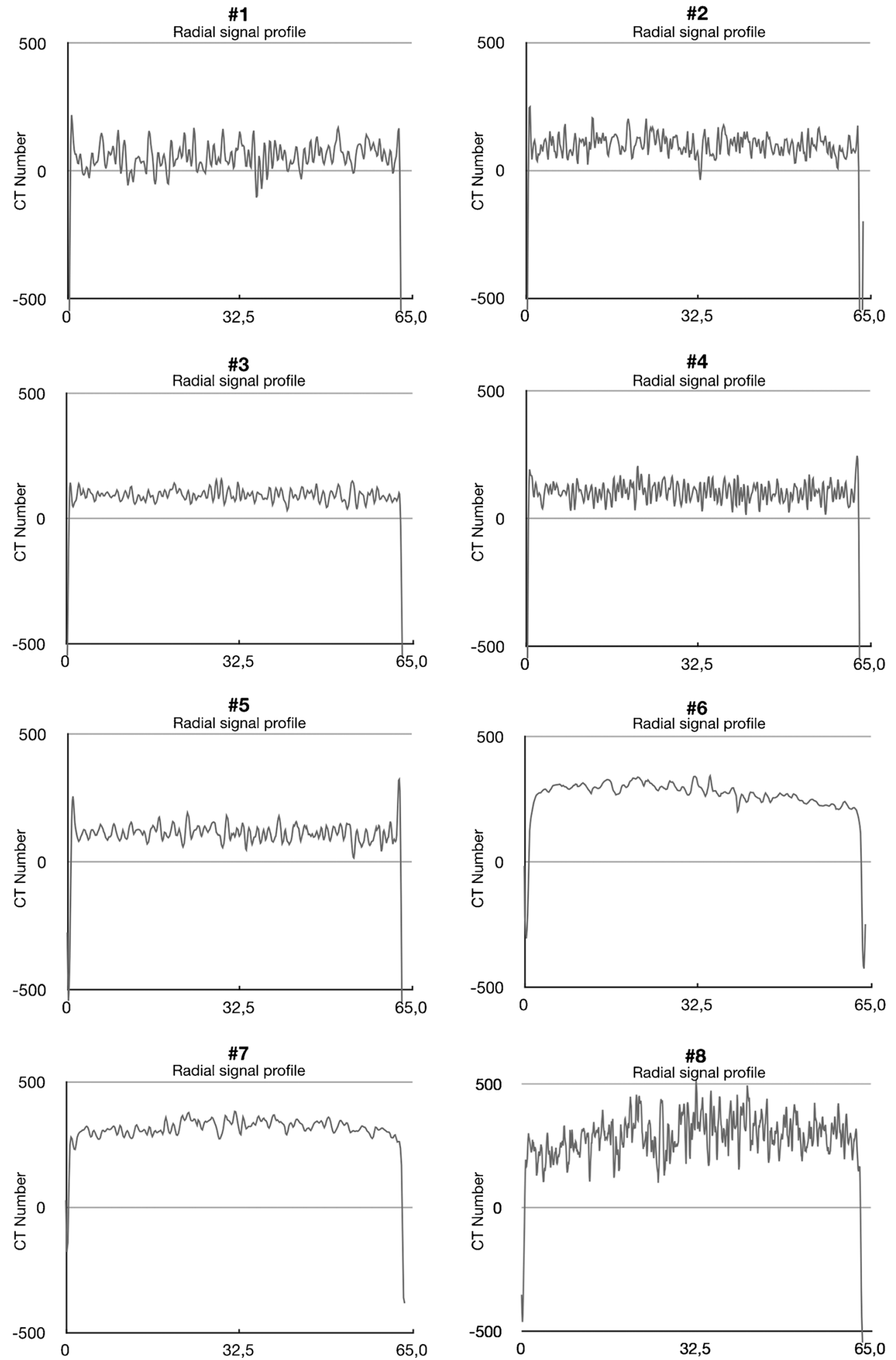
anatomical regions are given in Table 4. These results show that the trained users stated that acquisitions \#2 and \#4 stand out from the others by systematically taking the lead in ranking. The ranking of the other acquisitions varies considerably from one expert to another, which precludes classifying them. In general, we observed that the CBCT acquisitions (\#6 to \#8) showed better scores in the dentomaxillary region than in the temporal one (Table 4).
Fig. 5 Axial slice at the same position of the dental region

obtained with each protocol (\#1 to \#8; see Table 1) on the human dry skull. Window level: 700; window width: 4000

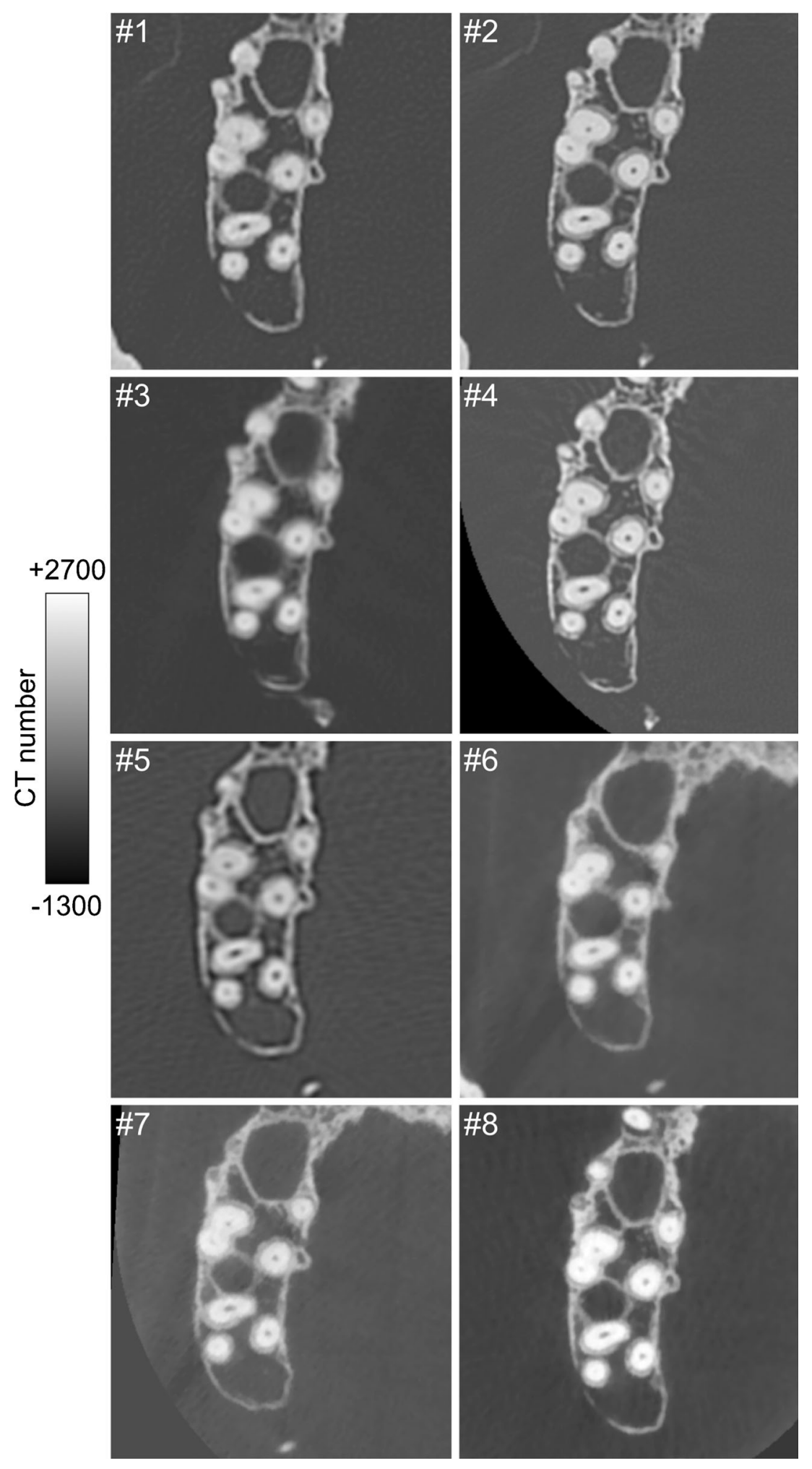




\section{Discussion}

CT models and methods

This study compares different high-resolution protocols between recent MSCT systems and one CBCT system. To our knowledge, our work is the first study that compares apparatuses of the same generation with a dedicated, sized phantom. In fact, previous studies did not use objective comparison methods with small FOV adapted phantoms [12, 13], or did not compare devices of the same generation or high-resolution protocols [14].
Fig. 6 Axial slice at the same position of the temporal region obtained with each protocol (\#1 to \#8; see Table 1) on the human dry skull. Window level: 700; window width: 4000

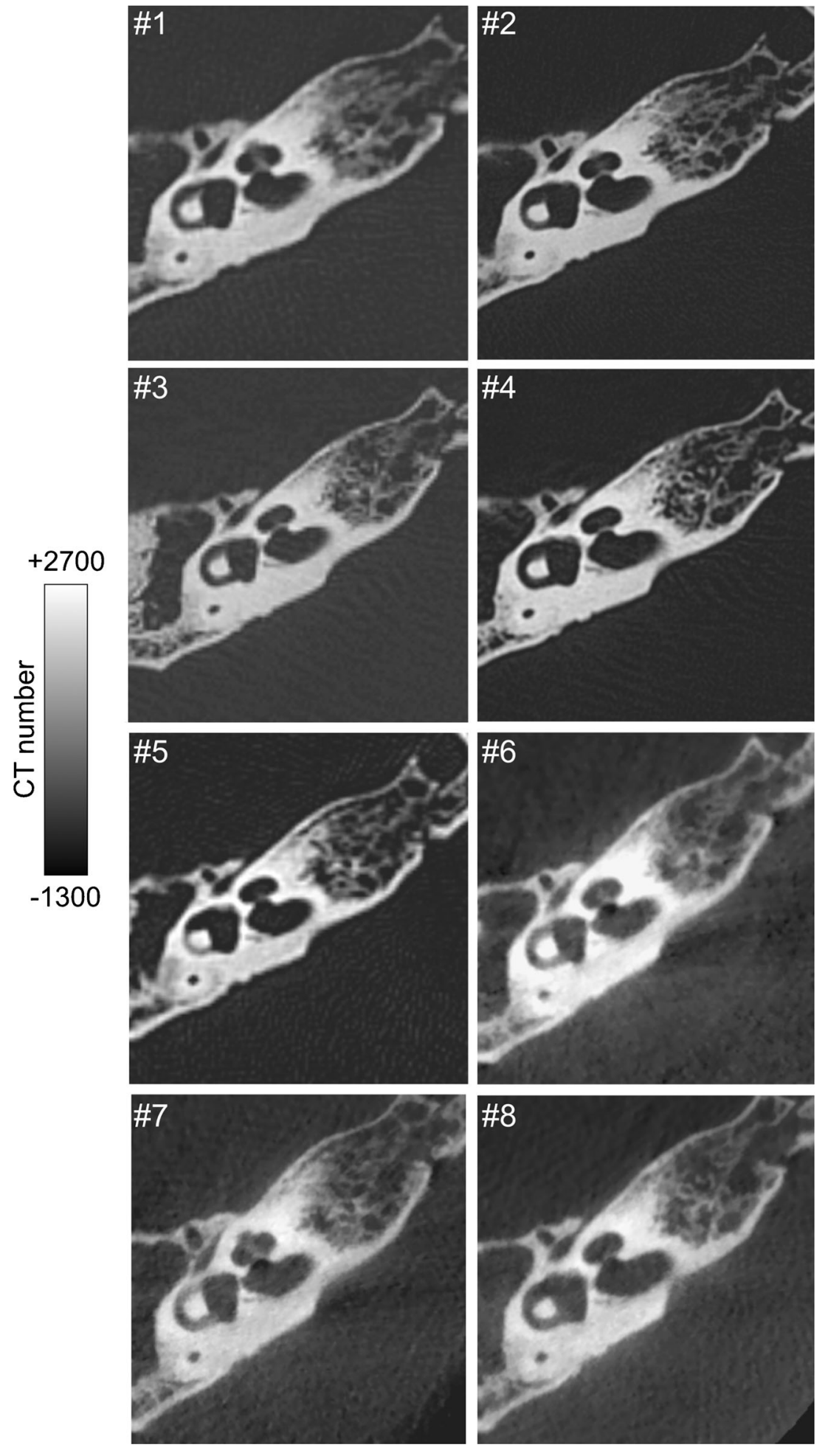


Phantom and dry-skull analysis

Our results do not demonstrate the supremacy of CBCT for bone-skull imaging that has been related in several studies [12, 15]. Our results, coming both from a phantom as well as a dry skull, demonstrate that MSCT protocols \#2 and \#4 outperformed all the other MSCT and CBCT protocols (Figs. 2, 3, 5, 6, Table 5). However, the CBCT acquisition \#8 provides interesting results. It is the only protocol showing the coil plate at $2.5 \mathrm{lp} \cdot \mathrm{mm}^{-1}$ on the phantom. It was ranked at the third position on the dento-maxillary analysis. This confirms that CBCT systems can be considered as a good alternative to MSCT in dentistry.

These results strengthen the expectations raised in an earlier study [16], and illustrate that spatial resolution is a complex issue that depends on both detectors' characteristics (unitary elements number and size), geometrical aspects (focal spot size, beam collimation), and acquisition mode (incremental, helical, number of projections, rotation duration time), but also reconstruction parameters, like: kernel type, pixel reconstructed size and reconstruction algorithm. However, as in other studies $[13,14]$, the phantom used does not allow a measure of resolution in the $\mathrm{z}$ direction.

Image homogeneity and geometric accuracy

The CBCT offers a large z-direction acquisition field by using an FPD. This geometry presents the advantage of acquiring a whole volume in a single rotation. However, it is more sensitive to scattered radiation, which has an impact on image homogeneity $[13,17]$, as demonstrated in our experience (Fig. 4). In order to limit the consequences of the Compton effect, we decided to realize acquisitions on a small FOVadapted phantom (Fig. 1) and on a dry skull (Figs. 5, 6). In terms of linearity and geometric accuracy, all systems present similar and robust results (Table 5).

\section{Practical aspect}

Our study is not exhaustive and is limited to the latest manufacturer models available at our university hospital. We also do not discuss other practical aspects, like the reconstruction speed and post-processing software. We note that, in our clinical practice, $\mathrm{CBCT}$ remains more accessible and available than MSCT systems. However, closer attention needs to be given to the management of patient motion artefacts in CBCT, due to rotation duration time (Table 1). For this reason, MSCT remains preferred in our practice in cases of bone trauma [1] and in the case of restless patients, especially in paediatrics. This additional point, which has not been evaluated in this work, is not in favour of CBCT. In this paper, we chose to limit our study to image properties and did not look at radiation safety considerations. Different kinds of studies state that MSCT is more irradiative than CBCT $[15,18]$.

State of the art

In general, MSCT companies are well established in the field of CT imaging and have been so since the beginning, therefore having more experience than companies developing CBCT systems. Today, CBCT is still quite a new technology in the field of clinical bone imaging, and our results confirm that currently it does not surpass MSCT. However, CBCT technology is still evolving, and current studies are trying to solve some problems such as homogeneity, which could impact the spatial resolution and improve the visualization of soft tissue $[13,17,19]$.

Acknowledgments Authors are grateful to Drs J.-L. Dietemann, A. Gangi, M. Ohana, C. Roy, F. Veillon, for access to the different devices. The authors gratefully acknowledge all technologists from the University Hospital of Strasbourg who participated in this study. Authors warmly thank General Electric for the loan of the phantom used in this study.

The scientific guarantor of this publication is P.Choquet. The authors of this manuscript declare no relationships with any companies, whose products or services may be related to the subject matter of the article. The authors state that this work has not received any funding. No complex statistical methods were necessary for this paper. Institutional Review Board approval was not required because no living subjects were used in this study.

\section{References}

1. Desikan RS, James YC (2014) Imaging of temporal bone trauma. Oper Tech Otolaryngol Head Neck Surg 25:110-117

2. Fuchs TO, Kachelriess M, Kalender WA (2000) System performance of multislice spiral computed tomography. IEEE Eng Med Biol Mag 19:63-70

3. Mahesh M, Cody DD (2007) Physics of cardiac imaging with multiple-row detector CT. Radiographics 27:1495-1509

4. Hassan A, Nazir SA, Alkaghi H (2011) Technical challenges of coronary CT angiography: today and tomorrow. Eur J Radiol 79: $161-171$

5. Miracle AC, Mukherji SK (2009) Conebeam CT of the head and neck, part 1: physical principles. Am J Neuroradiol 30:1088-1095

6. Miracle AC, Mukherji SK (2009) Conebeam CT of the head and neck, part 2: clinical applications. Am J Neuroradiol 30:1285-1292

7. Du LY, Umoh J, Nikolov NH, Pollmann SI, Lee TY, Holdsworth DW (2007) A quality assurance phantom for the performance evaluation of volumetric micro-CT systems. Phys Med Biol 52:7087-7108

8. Droege RT (1983) A quality assurance protocol for CT scanners. Radiology 146:244-246

9. Droege RT, Morin RL (1982) A practical method to measure the MTF of CT scanners. Med Phys 9:758-760

10. Holdsworth DW, Drangova M, Fenster A (1993) A high-resolution XRII-based quantitative volume CT scanner. Med Phys 20:449-462

11. Le Minor JM, Billmann F, Vetter JM, Ludes B (2009) Anatomie(s) \& pathologie(s): Les collections morphologiques de la faculté de médecine de Strasbourg, 1st edn. ID L'Édition, Bernardswiller 
12. Liang X, Jacobs R, Hassan B, Li L, Pauwels R, Corpas L, Souza PC, Martens W, Shahbazian M, Alonso A, Lambrichts I (2010) A comparative evaluation of Cone Beam Computed Tomography (CBCT) and Multi-Slice CT (MSCT) part I. On subjective image quality. Eur J Radiol 75:265-269

13. Carrino JA, Al Muhit A, Zbijewski W, Thawait GK, Stayman JW, Packard N, Senn R, Yang D, Foos DH, Yorkston J, Siewerdsen JH (2014) Dedicated cone-beam CT system for extremity imaging. Radiology 270:816-824

14. Pauwels R, Beinsberger J, Stamatakis H, Tsiklakis K, Walker A, Bosmans H, Bogaerts R, Jacobs R, Horner K (2012) Comparison of spatial and contrast resolution for cone-beam computed tomography scanners. Oral Surg Oral Med Oral Pathol Oral Radiol 114: $127-135$

15. Hofmann E, Schmid M, Sedlmair M, Banckwitz R, Hirschfelder U, Lell M (2014) Comparative study of image quality and radiation dose of cone beam and low-dose multislice computed tomography-an invitro investigation. Clin Oral Investig 18:301-311

16. Watanabe H, Honda E, Tetsumura A, Kurabayashi T (2011) A comparative study for spatial resolution and subjective image characteristics of a multi-slice CT and a cone-beam CT for dental use. Eur J Radiol 77:397-402

17. Dong X, Petrongolo M, Niu T, Zhu L (2013) Low-dose and scatterfree cone-beam CT imaging using a stationary beam blocker in a single scan: phantom studies. Comput Math Methods Med 2013:637614

18. Loubele M, Bogaerts R, Van Dijck E, Pauwels R, Vanheusden S, Suetens P, Marchal G, Sanderink G, Jacobs R (2009) Comparison between effective radiation dose of CBCT and MSCT scanners for dentomaxillofacial applications. Eur J Radiol 71:461-468

19. Reitz I, Hesse BM, Nill S, Tücking T, Oelfke U (2009) Enhancement of image quality with a fast iterative scatter and beam hardening correction method for kV CBCT. Z Med Phys 19:158-172 